\title{
Mechanism of $\mathrm{NO}_{x}$ sensing on $\mathrm{WO}_{3}$ surface : first principle calculations
}

\author{
Lama Saadi, ${ }^{\mathrm{a}}$, Caroline Lambert-Mauriat, ${ }^{\mathrm{a}}$, Vincent Oison, ${ }^{\mathrm{a}}$, Hela Ouali ${ }^{\mathrm{b}}$, \\ and Roland Hayn ${ }^{\mathrm{a}}$ \\ ${ }^{a}$ Aix-Marseille Université, Université de Toulon, CNRS, IM2NP UMR 7334, 13397 \\ Marseille Cedex 20, France \\ ${ }^{b}$ IPEST, Faculté de Tunis, La Marsa, Tunis, Tunisia
}

\begin{abstract}
Computational study of $\mathrm{NO}_{2}$ sensing on the $\mathrm{WO}_{3}$ (001) surface is presented. Our ab initio calculations reveal a two-step process of $\mathrm{NO}_{2}$ detection on the $\mathrm{WO}_{3}$ surface. In a first step the $\mathrm{NO}_{2}$ molecule is dissociated at an oxygen vacancy site, but a NO molecule remains adsorbed. In a second step NO is re-oxidized into $\mathrm{NO}_{2}$ by $\mathrm{O}_{2}$ of the surrounding air leading to the resistance increase which is experimentally observed. We also calculate the adsorption energy of $\mathrm{NO}$ on stoichiometric and non-stoichiometric $\mathrm{WO}_{3}$ surfaces and propose a method for the NO detection.
\end{abstract}

Keywords: $\mathrm{WO}_{3}$ surface, gas sensor, ab initio calculations, $\mathrm{NO}_{2}$ detection

\section{Introduction}

For many years, sensor devices based on metal-oxide semiconductors have been developed. The increasing interest in these devices comes from their low cost, easy microelectronic integration, sensitivity and short response time. Among the metal-oxides, $\mathrm{SnO}_{2}$ has been the first one used as sensing layer in such devices, in particular for the detection of CO [1]. Since tungsten trioxide, $\mathrm{WO}_{3}$, has proved that it could be a good alternative to $\mathrm{SnO}_{2}$, particularly for the ozone detection [2]. But the main disadvantage of these sensors is their lack of selectivity. Indeed these devices are based on the variation of the electrical resistivity of the metal-oxide in the presence of gas, which depends on both the oxido-reductor behavior of the target gas and the doping of the semiconductor. In the case of the n-type semiconductor $\mathrm{WO}_{3}$, an oxidizing gas increases the resistivity, whereas a reducing gas decreases it. To improve 
the selectivity of gas sensors, a better understanding of the gas adsorption mechanism is needed, in particular at the atomic scale. Therefore numerical simulations based on the density functional theory (DFT) are carried out in order to study the interaction of $\mathrm{WO}_{3}$ with different gases.

At room temperature, the crystallographic structure of $\mathrm{WO}_{3}$ is monoclinic (space group $\mathrm{P} 2{ }_{1} / \mathrm{c}$ ). This structure contains eight $\mathrm{WO}_{3}$ per unit cell, each $\mathrm{W}$ atom being six-fold coordinated by oxygen atoms. The most stable surface is found to be the $(\sqrt{2} \times \sqrt{2}) \mathrm{R} 45^{\circ}$ reconstructed (001) one, both by semiempirical simulations [3] and by DFT calculations [4]. This reconstruction is obtained by removing one oxygen atom row on two in the [110] direction. In addition it was confirmed that oxygen vacancies play a crucial role in the n-doping of the material, and consequently in the detection of $\mathrm{O}_{3}$ and $\mathrm{CO}$ molecules [5]: $\mathrm{O}_{3}$ is reduced to $\mathrm{O}_{2}$ decreasing the number of oxygen vacancies, whereas $\mathrm{CO}$ is oxidized to $\mathrm{CO}_{2}$ increasing the number of oxygen vacancies. Thus the oxygen stoichiometry of the surface should be a way to select the oxidizing or the reducing gas to be detected. In order to spatially separate oxygen vacancies, doubling the cell in the two perpendicular directions to the surface is necessary, and leads to an oxygen vacancy concentration at the surface of $\mathrm{C}_{v}=1 / 8$.

In continuation of our previous works, here we present computational results of the adsorption of $\mathrm{NO}_{2}$ molecules on the (001) $\mathrm{WO}_{3}$ surface. A selfconsistent first-principle SIESTA method carrying out geometry optimizations is used. Both the local-density approximation (LDA) and the generalized gradient approximation (GGA) functionals are considered.

This paper is organized as follows. Computational details are presented in section 2. The completeness of the basis sets used in the present SIESTA simulations is validated on characteristics of $\mathrm{NO}$ and $\mathrm{NO}_{2}$ (atomic distances, ionization potential, electron affinity and binding energies): results are compared to experiments and previous calculations. Then adsorption of $\mathrm{NO}_{2}$ molecule on the $\mathrm{WO}_{3}$ surface is studied in section 3: a two-step process is revealed. We also investigate the adsorption of $\mathrm{NO}$ molecule on $\mathrm{WO}_{3}$. Finally we conclude through a short discussion and a method to detect $\mathrm{NO}$ molecules is proposed in section 4 .

\section{Computational details}

Ab initio calculations are carried out using the SIESTA code $[6,7]$. The exchange-correlation potential is treated either within the local density ap- 
proximation (LDA) using the Ceperley-Alder functional [8] or within the generalized gradient approximation (GGA) using the Perdew-Burke-Ernzerhof functional [9]. In all calculations spin polarization is allowed and core electrons are treated within the frozen core approximation using norm-conserving Troullier-Martins [10] pseudopotentials. In addition, nonlinear core corrections are included in pseudopotentials for $\mathrm{W}, \mathrm{O}$ and $\mathrm{N}$ atoms. The wave function of the valence electrons (i.e. $2 s^{2} 2 p^{4}$ for $\mathrm{O}, 6 s^{2} 5 d^{4}$ for $\mathrm{W}$ and $2 s^{2} 2 p^{3}$ for $\mathrm{N}$ ) is expanded on a localized basis set consisting in finite-range pseudo-atomic orbitals [11]. A double- $\zeta$ basis set including orbitals of higher l-momentum to allow polarization is used for each atom (i.e. $3 d$ orbital for $\mathrm{O}$ and $\mathrm{N}$ and $6 p$ orbital for $\mathrm{W})$. The $\mathrm{W}$ and $\mathrm{O}$ basis sets are imported from our previous work [12]. The cutoff radii for orbitals localized on nitrogen atoms are: 5.64 and 3.03 a.u. for the $2 s$ orbitals, 7.25 and 2.85 a.u. for the $2 p$ orbitals and 3.65 a.u. for the $3 d$ orbital.

All calculations are performed using periodic supercells, which consist in a slab of several atomic planes and a thickness of vacuum of about $15 \AA$ along the perpendicular direction to the surface. The slab is built from the atomic structure of the $\gamma$-monoclinic phase with lattice parameters obtained from previous LDA (GGA) calculations: $a=7.24 \AA, b=7.45 \AA, c=7.61 \AA$ and $\beta=90.5^{\circ}\left(a=7.48 \AA, b=7.67 \AA, c=7.85 \AA\right.$ and $\left.\beta=90.4^{\circ}\right)$. Along the [001] direction, mixed WO planes alternate with pure O planes. As previously shown a good convergence with respect to the surface properties is reached for slabs containing $4 \mathrm{WO}$ planes [4]. In addition, we consider an oxygen vacancy concentration at the surface of $\mathrm{C}_{v}=1 / 8$, which implies doubling the cell in both $\mathbf{a}$ and $\mathbf{b}$ directions. This oxygen vacancy concentration is a good compromise between a good description of the physico-chemical mechanisms and the computation time. In all cases, the $\mathbf{k}-$ point sampling consists in a $4 \times 4 \times 1$ mesh-grid: four $\mathbf{k}$-points along $\mathbf{a}^{*}$ and $\mathbf{b}^{*}$ and one along $\mathbf{c}^{*}$.

\begin{tabular}{ccccccc}
\hline & & $\mathrm{d}_{\mathrm{N}=\mathrm{O}}(\AA)$ & $\widehat{\mathrm{ONO}}(\mathrm{deg})$. & E.A $(\mathrm{eV})$ & I.P. $(\mathrm{eV})$ & $\mathrm{E}_{\text {bond }}(\mathrm{eV})$ \\
\hline \hline \multirow{2}{*}{ This work } & LDA & 1.23 & 133.1 & 1.89 & 10.13 & 4.83 \\
& GGA & 1.24 & 132.8 & 1.51 & 10.13 & 3.40 \\
Experiments [13] & & 1.197 & 134.3 & 2.3 & 9.586 & 3.17 \\
\hline
\end{tabular}

Table 1: Chemical characteristics of isolated molecule $\mathrm{NO}_{2}$ calculated within LDA and GGA approximations. 
In order to validate the completeness of the basis sets, electron affinity (E.A.), ionization potential (I.P.), binding energies and structural geometry are calculated for $\mathrm{NO}$ and $\mathrm{NO}_{2}$ molecules (see tables 1 and 2). In comparison with experiments, the calculations slightly overestimate the length of NO bonds whatever functional and molecule. Binding energies and ionization potential are better estimated by the GGA. On the contrary, electron affinity is systematically highly underestimated. Let us note that previous theoretical works based on the DFT showed the same trends $[14,15]$. More recent semiempirical works have increased the accuracy with experiments [16].

\begin{tabular}{lccccc}
\hline & & $\mathrm{d}_{\mathrm{N}=\mathrm{O}}(\AA)$ & E.A $(\mathrm{eV})$ & I.P. $(\mathrm{eV})$ & $\mathrm{E}_{\text {bond }}(\mathrm{eV})$ \\
\hline \hline \multirow{2}{*}{ This work } & LDA & 1.18 & 0.014 & 10.26 & 7.6 \\
Experiments [13] & GGA & 1.19 & 0.017 & 10.16 & 6.6 \\
& & 1.15 & 0.026 & 9.26 & 6.3 \\
\hline
\end{tabular}

Table 2: Chemical characteristics of isolated molecule NO calculated within LDA and GGA approximations.

\section{Results}

\section{1. $\mathrm{NO}_{2}$ adsorption}

As for $\mathrm{O}_{3}$ molecule [5], oxygen vacancy at the surface is supposed to be the preferential site for the chemisorption of the $\mathrm{NO}_{2}$ molecule. Our calculations show that the $\mathrm{NO}_{2}$ molecule remains in weak interaction with the surface, when the $\mathrm{N}$ atom of the molecule is presented on top of a $\mathrm{W}_{\mathrm{V}}$ oxygen vacancy site. The resulting adsorption energy, $\mathrm{E}_{\mathrm{ads}}$, is almost zero and the charge transfer from the surface to the molecule is weak (0.079 electrons in GGA). Such a behavior is expected since $\mathrm{NO}_{2}$ molecule is polar along the single $\mathrm{N}-\mathrm{O}$ bond, with an electrical dipole moment of $0.316 \mathrm{D}$ [13]. In the opposite, when one of the two atoms (noted $\mathrm{O}_{\mathrm{s}}$ ) of the $\mathrm{NO}_{2}$ molecule is presented on top of a $\mathrm{W}_{\mathrm{V}}$ site, the $\mathrm{NO}_{2}$ molecule is dissociated as shown in figure 2. In comparison with the geometry of the isolated $\mathrm{NO}_{2}$ molecule (see table 1), the $\mathrm{N} \cdots \mathrm{O}_{\mathrm{s}}$ distance drastically increases from $1.23 \AA$ to $1.58 \AA(2.18 \AA)$ within the LDA (GGA), whereas the second NO distance decreases to $1.17 \AA$, and the $\widehat{\mathrm{O}_{\mathrm{s}} \mathrm{NO}}$ angle reaches $110^{\circ}$ for both the functionals. These results indicate that the $\mathrm{NO}_{2}$ molecule is almost dissociated. Thus the detection mechanism 
a)

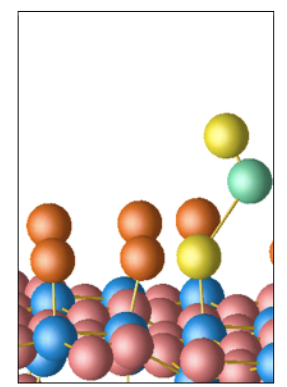

b)

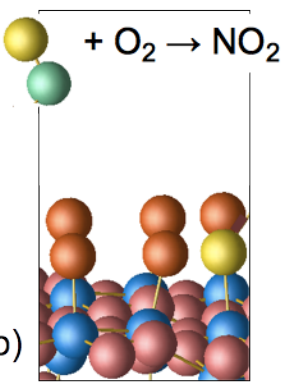

Figure 1: Dissociation of $\mathrm{NO}_{2}$ on a $\mathrm{W}_{\mathrm{V}}$ oxygen vacancy site: a) NO remains in interaction with the surface (first step) ; b) $\mathrm{NO}$ is re-oxidized into $\mathrm{NO}_{2}$ by $\mathrm{O}_{2}$ of the atmosphere (second step). Oxygen atoms of the $\mathrm{NO}_{\mathrm{x}}$ molecules are yellow, whereas $\mathrm{N}$ atom is green. In the slab, oxygen atoms are red and tungsten atoms are blue.

of $\mathrm{NO}_{2}$ on $\mathrm{W}_{\mathrm{V}}$ involves the oxidation of the surface, leading to the filling of an oxygen vacancy and to the release of a NO molecule in gas phase. The balance equation can be written as:

$$
\mathrm{W}_{\mathrm{n}} \mathrm{O}_{3 \mathrm{n}-1}+\mathrm{V}_{\mathrm{O}}^{\bullet}+\mathrm{e}^{\prime}+\mathrm{NO}_{2}^{\text {gas }} \longrightarrow \mathrm{W}_{\mathrm{n}} \mathrm{O}_{3 \mathrm{n}}+\mathrm{NO}^{\text {gas }},
$$

where $\mathrm{W}_{\mathrm{n}} \mathrm{O}_{3 \mathrm{n}-1}$ corresponds to the oxygen deficient surface and $\mathrm{W}_{\mathrm{n}} \mathrm{O}_{3 \mathrm{n}}$ to the stoichiometric one. The corresponding reaction enthalpy, $\Delta \mathrm{H}$ (see table 3 ), which is obtained from supercell calculations by

$$
\Delta \mathrm{H} \approx \mathrm{E}\left(\mathrm{W}_{\mathrm{n}} \mathrm{O}_{3 \mathrm{n}}\right)+\mathrm{E}(\mathrm{NO})-\mathrm{E}\left(\mathrm{W}_{\mathrm{n}} \mathrm{O}_{3 \mathrm{n}-1}\right)-\mathrm{E}\left(\mathrm{NO}_{2}\right)
$$

amounts to $-0.47 \mathrm{eV}(-0.50 \mathrm{eV})$ in LDA (GGA). When interaction between the released NO molecule and the surface is taken into account, chemisorption energy becomes

$$
\mathrm{E}_{\mathrm{chem}} \approx \Delta \mathrm{H}+\mathrm{E}_{\mathrm{ads}},
$$

where $\mathrm{E}_{\mathrm{ads}}$ is the adsorption energy between $\mathrm{NO}$ and the surface defined as follows:

$$
\mathrm{E}_{\mathrm{ads}}=\mathrm{E}\left(\mathrm{W}_{\mathrm{n}} \mathrm{O}_{3 \mathrm{n}}+\mathrm{NO}\right)-\mathrm{E}\left(\mathrm{W}_{\mathrm{n}} \mathrm{O}_{3 \mathrm{n}}\right)-\mathrm{E}(\mathrm{NO}) .
$$

The characteristics of the NO adsorption are given in table 5. Thus the LDA gives an adsorption energy of $-1.12 \mathrm{eV}$, which is the double of the GGA value $(-0.55 \mathrm{eV})$. In all cases the adsorption mechanism involves a charge transfer (CT) from the molecule to the surface. Let us note that CT is slightly higher within GGA $\left(0.27 \mathrm{e}^{-}\right)$than within LDA $\left(0.21 \mathrm{e}^{-}\right)$. Thus the interaction 


\begin{tabular}{ccc}
\hline & $\Delta \mathrm{H}$ & $\mathrm{E}_{\text {chem }}$ \\
\hline \hline LDA & -0.47 & -1.59 \\
GGA & -0.50 & -1.05 \\
\hline
\end{tabular}

Table 3: Reaction enthalpy, $\Delta \mathrm{H}$, for the $\mathrm{NO}_{2}$ dissociation on $\mathrm{W}_{V}$ following equation 1 and chemisorption energy of $\mathrm{NO}_{2}$ taking into account the interaction of the released NO molecule with the surface. Values are given in $\mathrm{eV}$.

between $\mathrm{NO}$ and $\mathrm{O}_{\mathrm{s}}$ can be described as a "closed-shell" ionic interaction characterized by a notable $\mathrm{CT}$ and a large $\mathrm{NO}_{\mathrm{s}}$ distance within GGA. In the contrary, the interaction is more covalent within the LDA: the CT is smaller and the $\mathrm{NO}_{\mathrm{s}}$ distance is quite shorter. The discrepancy between the two functionals is due to the well-known overbinding (underbinding) of $\mathrm{NO}$ with the surface when using the LDA (GGA).

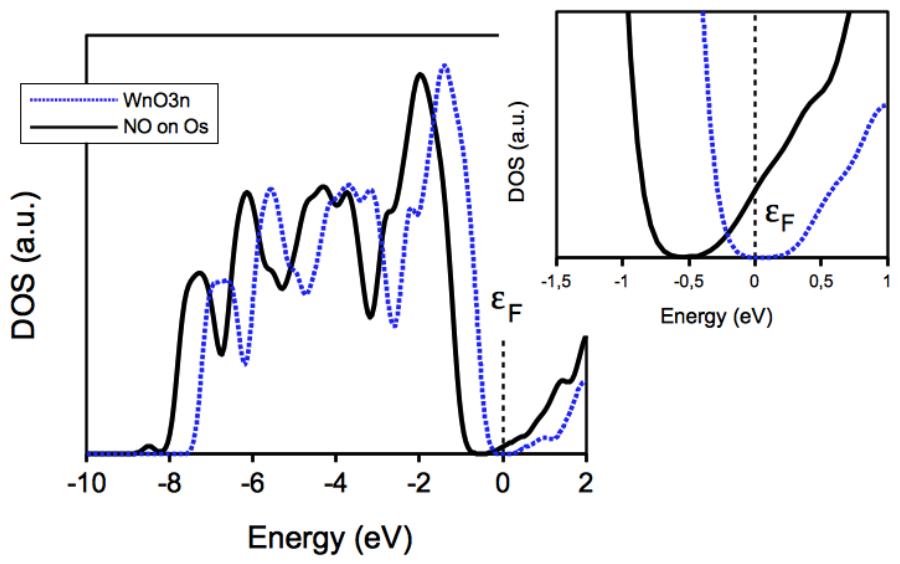

Figure 2: Calculated total density of states of stoichiometric surfaces: comparison between isolated surface (dashed line) and surface with $\mathrm{NO}$ adsorbed on an $\mathrm{O}_{\mathrm{s}}$ oxygen atom (full line). The levels of Fermi, $\epsilon_{\mathrm{F}}$, are shifted to the reference energy $0 \mathrm{eV}$. LDA results.

The calculated DOS highligths an n-doping when the NO molecule remains in interaction with the sensitive layer (Fig. 2). This is due to the charge transfer from the molecule to the surface. The same trend was shown for the non-stoichiometric surface with an oxygen vacancy [12]. In comparison with 
the DOS of the stoichiometric (001) $\mathrm{WO}_{3}$ surface, the Fermi level is shifted towards the conduction band. Thus the $\mathrm{NO}_{2}$ dissociation should not induce a resistivity increase during the gas exposure, as observed experimentally [17, $18,19,20]$, if the released NO molecule remains adsorbed on the surface. But

\begin{tabular}{cccccccc}
\hline & $\mathrm{d}_{\mathrm{WO}}$ & $\mathrm{d}_{\mathrm{NO}_{\mathrm{s}}}$ & $\mathrm{d}_{\mathrm{NO}}$ & $\widehat{\mathrm{WON}}$ & $\widehat{\mathrm{O}_{\mathrm{s}} \mathrm{NO}}$ & $\mathrm{E}_{\mathrm{ads}}$ & $\mathrm{CT}$ \\
\hline \hline LDA & 1.89 & 1.58 & 1.17 & 119 & 111 & -1.12 & -0.208 \\
GGA & 1.75 & 2.18 & 1.17 & 134 & 108 & -0.55 & -0.273 \\
\hline
\end{tabular}

Table 4: Characteristics of the NO adsorption on oxygen atom of the surface, noted $\mathrm{O}_{\mathrm{s}}$. Distances are given in $\AA$, angles in degrees, adsorption energy $\mathrm{E}_{\text {ads }}$ in $\mathrm{eV}$ and the charge transfer CT from NO to the surface in electrons (minus sign in CT means that NO loses electrons). Comparison between LDA and GGA.

when considering the recombination of the released NO with an atmospheric $\mathrm{O}_{2}$ molecule, following the reaction:

$$
\mathrm{NO}^{\text {ads }}+\frac{1}{2} \mathrm{O}_{2} \longrightarrow \mathrm{NO}_{2}^{\text {gas }}
$$

whose enthalpy is $-1.08 \mathrm{eV}(-0.87 \mathrm{eV})$ in LDA (GGA), the total reaction can be written as:

$\mathrm{W}_{\mathrm{n}} \mathrm{O}_{3 \mathrm{n}-1}+\mathrm{V}_{\mathrm{O}}^{\bullet}+\mathrm{e}^{\prime}+\mathrm{NO}_{2}^{\text {gas }}+\frac{1}{2} \mathrm{O}_{2}^{\text {gas }} \longrightarrow\left[\mathrm{W}_{\mathrm{n}} \mathrm{O}_{3 \mathrm{n}}+\mathrm{NO}^{\text {ads }}+\frac{1}{2} \mathrm{O}_{2}^{\text {gas }}\right]^{*} \longrightarrow \mathrm{W}_{\mathrm{n}} \mathrm{O}_{3 \mathrm{n}}+\mathrm{NO}_{2}^{\text {gas }}$

with a transition state

$$
\mathrm{W}_{\mathrm{n}} \mathrm{O}_{3 \mathrm{n}}+\mathrm{NO}^{\text {ads }}+\frac{1}{2} \mathrm{O}_{2}^{\text {gas }}
$$

Thus, the mechanism of $\mathrm{NO}_{2}$ detection involves two steps: i) the $\mathrm{NO}_{2}$ molecule is first dissociated, leading to the annihilation of an oxygen vacancy. The released NO molecule remains in interaction with the surface. The recombination of the oxygen vacancy is then electrically balanced by the charge transfer from the adsorbed NO to the surface and no modification in the electrical resistance should be expected; ii) in a second step, NO is re-oxidized into $\mathrm{NO}_{2}$ by $\mathrm{O}_{2}$ of the atmosphere. Finally the surface is partially oxidized, decreasing the oxygen vacancy concentration and consequently the n-doping of the material. In that process the life time of the transition state (Eq. 7) is 
supposed to be extremely short in comparison to the time of oxygen vacancy formation. After $\mathrm{NO}_{2}$ flux is stopped, experimental recovery time is generally higher than the response one (see for example [18]). According to the process we describe the recovery time should be only due to the restoration of the initial oxygen vacancy concentration.

\subsection{NO adsorption}

The non-dissociative adsorption of the NO molecule on an oxygen deficient $\mathrm{W}_{\mathrm{V}}$ tungsten is also considered. In this case, the $\mathrm{N}$ atom is presented on top of the $\mathrm{W}_{\mathrm{V}}$ site, because NO molecule is known as ligand when the $\mathrm{N}$ atom is bonded with a metal atom. The energy adsorption, $\mathrm{E}_{\text {ads }}$, is calculated as follows:

$$
\mathrm{E}_{\mathrm{ads}}=\mathrm{E}\left(\mathrm{W}_{\mathrm{n}} \mathrm{O}_{3 \mathrm{n}-1}+\mathrm{NO}\right)-\mathrm{E}\left(\mathrm{W}_{\mathrm{n}} \mathrm{O}_{3 \mathrm{n}-1}\right)-\mathrm{E}(\mathrm{NO}),
$$

where $\mathrm{E}\left(\mathrm{W}_{\mathrm{n}} \mathrm{O}_{3 \mathrm{n}-1}\right)$ is the total energy of the sub-stoichiometric slab. As shown in table 5 , two equilibrium $\mathrm{N} \cdots \mathrm{W}_{\mathrm{V}}$ distances are found, corresponding to the two complexes (noted conf1 and conf2) that NO can form with a metal atom [21]. In the conf1 geometry, the molecule is linearly adsorbed

\begin{tabular}{llccccc}
\hline & & $\mathrm{d}_{\mathrm{W}_{\mathrm{V} \cdots \mathrm{N}}}$ & $\mathrm{d}_{\mathrm{NO}}$ & $\widehat{\mathrm{W}_{\mathrm{V}} \mathrm{NO}}$ & $\mathrm{E}_{\mathrm{ads}}$ & $\mathrm{CT}$ \\
\hline \hline \multirow{2}{*}{ conf1 } & LDA & 1.86 & 1.20 & 179 & -0.74 & -0.030 \\
& GGA & 1.97 & 1.20 & 174 & -0.24 & +0.018 \\
\hline \multirow{2}{*}{ conf2 } & LDA & 2.24 & 1.18 & 132 & -0.66 & -0.034 \\
& GGA & 2.44 & 1.19 & 126 & -0.22 & -0.057 \\
\hline \hline
\end{tabular}

Table 5: Characteristics of the NO adsorption on oxygen vacancy at the surface, $\mathrm{W}_{\mathrm{V}}$, for GGA and LDA approximations. Distances are given in $\AA$, angles in degrees and $\mathrm{E}_{\text {ads }}$ in $\mathrm{eV}$.

and the $\mathrm{N} \cdots \mathrm{W}_{\mathrm{V}}$ distance is short: $1.86 \AA(1.97 \AA)$ in LDA (GGA). In the conf2 geometry the $\mathrm{N} \cdots \mathrm{W}_{\mathrm{V}}$ distance is longer: $2.24 \AA(2.44 \AA)$ within the LDA (GGA), and the molecule is tilted. For a given functional, the value of adsorption energy is similar for the two configurations: the difference in adsorption energy between conf1 and conf2 is found to be $0.02 \mathrm{eV}(0.08 \mathrm{eV})$ in LDA (GGA). As expected, the adsorption interaction which includes a large part of dispersive effects is overbinded within the LDA and underbinded within the GGA. As shown on the calculated DOS (Fig. 3) an additional 


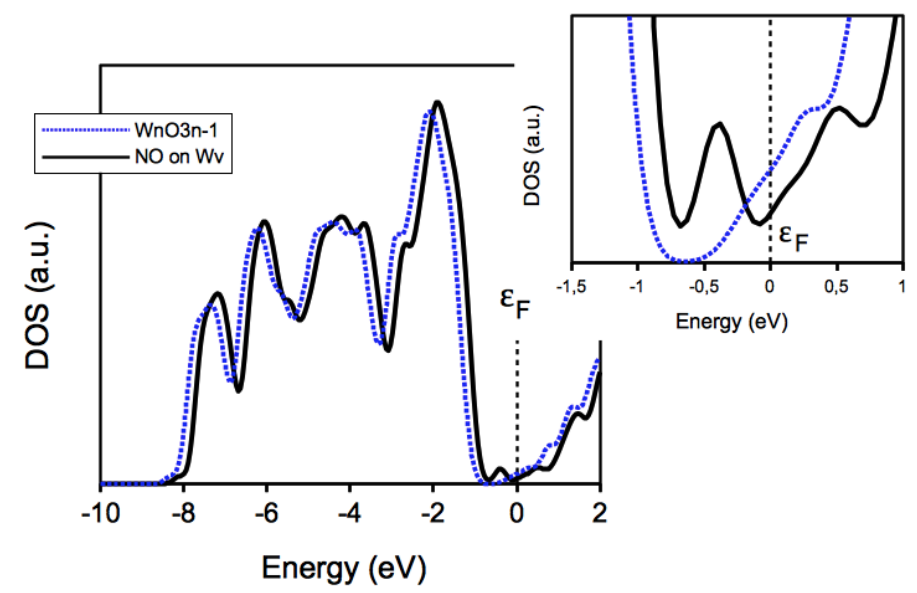

Figure 3: Calculated total density of states of sub-stoichiometric surfaces: comparison between isolated surface (dashed line) and surface in interaction with NO molecule in conf1 configuration (full line). The levels of Fermi, $\epsilon_{\mathrm{F}}$, are shifted to the reference energy $0 \mathrm{eV}$. LDA results.

state appears in the band gap due to the interaction of $\mathrm{NO}$ with the surface. Nevertheless the n-doping (i.e. the density of state at the level of Fermi) is a little weakened only. Consequently no major decrease should be noticed on the electrical resistivity.

\section{Discussion and conclusion}

In the present work we first show that $\mathrm{NO}_{2}$ is dissociated on an oxygen vacancy $\mathrm{W}_{\mathrm{V}}$ site with a reaction enthalpy of $0.5 \mathrm{eV}$ approximately whatever the exchange-correlation functional. The reaction involves the recombination of an oxygen vacancy with an oxygen atom of $\mathrm{NO}_{2}$ leading to the release of a $\mathrm{NO}$ molecule, which is quickly reoxidized into $\mathrm{NO}_{2}$ by dioxygen of the air. In poor $\mathrm{O}_{2}$ conditions, $\mathrm{NO}$ can remain adsorbed onto the surface with an adsorption energy reaching $1 \mathrm{eV}(0.5 \mathrm{eV})$ within LDA (GGA). Second, our calculations show that the adsorption of a $\mathrm{NO}$ molecule on an oxygen atom of the stoichiometric $\mathrm{WO}_{3}$ (001) surface leads to an n-type doping, which is very similar to the one observed when oxygen vacancy is created at the surface. Thus, the detection of NO molecules should be possible by using stoichiometric $\mathrm{WO}_{3}$ sensitive layer in gas sensor devices. The response should result in 
a decrease of the electrical resistivity, the opposite of $\mathrm{NO}_{2}$. Such a behaviour is already mentioned by Becker et al. for $\mathrm{SnO}_{2}$-based sensor [22] and by $\mathrm{Lu}$ et al. in sensors based on variation of the electromotive force [23]. It is noted that the $\mathrm{NO}$ response is always much smaller than the $\mathrm{NO}_{2}$ response. Nervertheless, the authors mention the oxidation of NO by atmospheric dioxygen before interacting with the sensitive layer [17, 24, 25, 18]. Consequently, the response of the sensor is characterized by the resistivity increase, but lower in amplitude in comparison with $\mathrm{NO}_{2}$. In conclusion, two major parameters should be taken into account for the detection of $\mathrm{NO}_{x}$ gases: i) the concentration of oxygen vacancies at the surface (i.e. the stoichiometry of $\mathrm{WO}_{3}$ sensitive layers); ii) the partial pressure of $\mathrm{O}_{2}$ in the ambient atmosphere. This last point is underlined by Akiyama et al. which shows that NO is detected only in presence of at least $1 \%$ of $\mathrm{O}_{2}$, whereas $\mathrm{NO}_{2}$ detection is almost independent of $\mathrm{O}_{2}$ concentration [20]. But as mentioned before only the $\mathrm{NO}_{2}$ molecules are detected because the NO molecules should be oxidized before interacting with the $\mathrm{WO}_{3}$ surface. Thus, our present results show a way to improve the detection of $\mathrm{NO}$ and $\mathrm{NO}_{2}$ molecules by $\mathrm{WO}_{3}$-based sensors.

We point to the importance of oxygen vacancy concentration at the surface, which can be easily controlled by deposition and annealing conditions. According to our results, a higher surface reduction (increase of surface vacancies) should lead to a high critical concentration of $\mathrm{NO}_{2}$ detection. In contrary, the stoichiometric surface should allow to detect $\mathrm{NO}$ but not $\mathrm{NO}_{2}$, in the absence of $\mathrm{O}_{2}$ in the surrounding atmosphere. These results have to be confirmed by experiments in a future work.

\section{Acknowledgment}

This work was granted access to the HPC resources of CINES under the allocation 2013-095073 made by GENCI.

\section{References}

[1] N. Bârsan, J. Stetter, M. Findlay, W. Göpel, High performance gas sensing of CO: comparative tests for $\left(\mathrm{SnO}_{2}\right.$-based) semiconducting and for electrochemical sensors, Sensors and Actuators B 66 (2000) 31.

[2] R. Boulmani, M. Bendahan, C. Lambert-Mauriat, M. Gillet, K. Aguir, Correlation between rf-sputtering parameters and $\mathrm{WO}_{3}$ sensor response towards ozone, Sensors and Actuators B 125 (2007) 622. 
[3] P. M. Oliver, S. C. Parker, R. G. Egdell, F. H. Jones, Computer simulation of the surface structures of $\mathrm{WO}_{3}$, J. Chem. Soc., Faraday Trans. 92 (1996) 2049.

[4] C. Lambert-Mauriat, V. Oison, L. Saadi, K. Aguir, Ab initio study of oxygen point defects on tungsten trioxide surface, Surface Science 606 (2012) 40-45.

[5] V. Oison, L. Saadi, C. Lambert-Mauriat, R. Hayn, Mechanism of CO and $\mathrm{O}_{3}$ sensing on $\mathrm{WO}_{3}$ surfaces: First principle study, Sensors and Actuators B: Chemical 160 (2011) 505.

[6] P. Ordejón, E. Artacho, J. M. Soler, Self-consistent order-n densityfunctional calculations for very large systems, Phys. Rev. B 53 (1996) R10441.

[7] J. M. Soler, E. Artacho, J. D. Gale, A. Garcia, J. Junquera, P. Ordejón, D. Sánchez-Portal, The siesta method for ab initio order-n materials simulation, J. Phys.: Condens. Matter 14 (2002) 2745.

[8] D. M. Ceperley, B. J. Alder, Ground state of the electron gas by a stochastic method, Phys. Rev. Lett. 45 (1980) 566.

[9] J. P. Perdew, K. Burke, M. Ernzerhof, Generalized gradient approximation made simple, Phys. Rev. Lett. 77 (1996) 3865.

[10] N. Troullier, J. L. Martins, Efficient pseudopotentials for plane-wave calculations, Phys. Rev. B 43 (1991) 1993.

[11] O. F. Sankey, D. J. Niklewski, Ab initio multicenter tight-binding model for molecular-dynamics simulations and other applications in covalent systems, Phys. Rev. B 40 (1989) 3979.

[12] C. Lambert-Mauriat, V. Oison, Density-functional study of oxygen vacancies in monoclinic tungsten oxide, J. Phys.: Condens. Matter 18 (2006) 7361.

[13] D. R. Lide, Handbook of chemistry and physics, 89th Edition, CRC, 2008, pp. 9-50. 
[14] P. Stampfuß, W. Wenzel, Accurate multireference calculations of the electron affinity of $\mathrm{NO}, \mathrm{BO}$ and $\mathrm{O}_{2}$, Chemical Physics Letters 370 (2003) 478-484.

[15] J. Prades, A. Cirera, J. Morante, J. Pruneda, P. Ordejón, Ab initio study of $\mathrm{NO}_{x}$ compounds adsorption on $\mathrm{SnO}_{2}$ surface, Sensors and Actuators B 126 (2007) 62.

[16] C. A. Arrington, T. H. Dunning, D. E. Woon, Electron affinity of NO, J. Phys. Chem. A 111 (44) (2007) 11185-11188.

[17] M. Penza, M. A. Tagliente, L. Mirenghi, C. Gerardi, C. Martucci, G. Cassano, Tungsten trioxide $\left(\mathrm{WO}_{3}\right)$ sputtered thin films for a $\mathrm{NO}_{x}$ gas sensor, Sensors and Actuators B 50 (1998) 9.

[18] T. Kida, A. Nishiyama, M. Yuasa, K. Shimanoe, N. Yamazoe, Highly sensitive $\mathrm{NO}_{2}$ sensors using lamellar-structured $\mathrm{WO}_{3}$ particles prepared by an acidification method, Sensors and Actuators B 135 (2009) 568.

[19] Y. Qin, M. Hu, J. Zhang, Microstructure characterization and $\mathrm{NO}_{2^{-}}$ sensing properties of tungsten oxide nanostructures, Sensors and Actuators B 150 (2010) 339.

[20] M. Akiyama, Z. Zhang, J. Tamaki, N. Miura, N. Yamazoe, Tungsten oxide-based semiconductor sensor for detection of nitrogen oxides in combustion exhaust, Sensors and Actuators B 14 (1993) 619.

[21] J. H. Enemark, R. D. Feltham, Principles of structure, bonding, and reactivity for metal nitrosyl complexes, Coord. Chem. Rev. 13 (1974) 339 .

[22] T. Becker, S. Mühlberger, C. B. v. Braunmühl, G. Müller, T. Ziemann, Air pollution monitoring using tin-oxide-based microreactor systems, Sensors and Actuators B 69 (2000) 108.

[23] G. Lu, N. Miura, N. Yamazoe, Stabilized zirconia-based sensors using $\mathrm{WO}_{3}$ electrode for detection of $\mathrm{NO}$ or $\mathrm{NO}_{2}$, Sensors and Actuators $\mathrm{B}$ 65 (2000) 125.

[24] D. Manno, A. Serra, M. D. Giulio, G. Micocci, A. Tepore, Physical and structural characterization of tungsten oxide thin films for NO gas detection, Thin Solid Films 324 (1998) 44. 
[25] B. Fruhberger, N. Stirling, F. G. Grillo, S. Ma, D. Ruthven, R. J. Lad, B. G. Frederick, Detection and quantification of nitric oxide in human breath using a semiconducting oxide based chemiresistive microsensor, Sensors and Actuators B 76 (2001) 226. 